\title{
BONE DEVELOPMENT AND FRACTURE HEALING IS NORMAL IN MICE THAT HAVE A DEFECT IN THE DEVELOPMENT OF THE LYMPHATIC SYSTEM
}

\section{A.L. McCarter, A. Khalid, Y. Yi, M. Monroy, H. Zhao, J.J. Rios, M.T. Dellinger}

Division of Surgical Oncology (ALM,AK,MM,MTD) Department of Surgery and the Hamon Center for Therapeutic Oncology Research, UT Southwestern Medical Center, Dallas; Center for Pediatric Bone Biology and Translational Research (AK,JJR), Scottish Rite for Children, Dallas; Biomedical Sciences (YY,HZ), Texas A\&M College of Dentistry, Dallas; McDermott Center for Human Growth and Development (JJR), Department of Pediatrics, Department of Orthopedic Surgery, and the Simmons Comprehensive Cancer Center, UT Southwestern Medical Center, Dallas; and Department of Molecular Biology and the Hamon Center for Regenerative Science and Medicine (MTD), UT Southwestern Medical Center, Dallas, TX, USA

\section{ABSTRACT}

Ectopic lymphatics form in bone and promote bone destruction in diseases such as Gorham-Stout disease, generalized lymphatic anomaly, and kaposiform lymphangiomatosis. However, the role lymphatics serve in normal bone development and repair is poorly understood. The objective of this study was to characterize bone development and fracture healing in mice that have a defect in the development of the lymphatic vasculature. We found that bones in wild-type adult mice and mouse embryos did not have lymphatics. We also found that bone development was normal in Vegfr $3^{\text {Chy/Chy }}$ embryos. These mice do not have lymphatics and die shortly after birth. To determine whether lymphatics serve a role in postnatal bone development and fracture healing, we analyzed bones from Vegfr $3^{\text {wt/Chy }}$ mice. These mice are viable and have fewer lymphatics than wild-type mice. We found that postnatal bone development and fracture healing was normal in Vegfr $3^{\text {wt/Chy }}$ mice. Taken together, our results suggest that lymphatics do not play a major role in normal bone development or repair.
Keywords: VEGFR3, lymphangiogenesis, bone development, fracture healing, GorhamStout disease

More than 2 million Americans break a bone each year (1). Most bone fractures heal uneventfully and individuals can go back to performing their normal activities after their cast has been removed. However, fractures fail to heal in approximately $5-10 \%$ of individuals with a broken bone (2). Non-union bone fractures are frequently treated with surgical procedures and bone grafts (2). Unfortunately, those treatments can be expensive and increase patient morbidity (2). Therefore, new safe and effective treatments are needed for non-union bone fractures. A better understanding of normal bone development and healing could lead to new pharmacologic treatments for patients with a non-union bone fracture.

Bone development occurs by two separate mechanisms (3). Flat bones, such as those of the skull, are formed by intramembranous ossification, whereas long bones, such as those of the limbs, are formed by endochondral 
ossification (3). During intramembranous ossification, mesenchymal cells differentiate into osteoblasts which create an osteoid matrix that is later mineralized $(3,4)$. During endochondral ossification, chondrocytes create a hyaline cartilage template (3-5). Perichondral cells then differentiate into osteoblasts which produce a layer of bone around the shaft of the cartilage template (3-5). During this time, chondrocytes in the center of the cartilage template become hypertrophic and stimulate the invasion of blood capillaries and osteoblasts (3-5). Osteoblasts start to produce trabecular bone within the cartilage template as osteoclasts begin to erode bone to create the marrow cavity $(3,4)$. As development proceeds, the activity of chondrocytes, osteoblasts, and osteoclasts causes bones to increase in length and diameter (5).

Bone is one of the few organs that can repair itself in response to injury. At the site of injury, severed blood vessels create a hematoma and cytokine-secreting inflammatory cells clear debris and dead cells $(6,7)$. As with bone development, intramembranous and endochondral ossification occur during fracture healing $(6,7)$. Intramembranous ossification takes place at the proximal ends of the fracture and endochondral ossification occurs inbetween the fractured ends to create a soft callus which stabilizes the fracture $(6,7)$. The soft callus becomes a hard callus as the cartilage is resorbed and replaced with bone $(6,7)$. Over time, the protruding hard callus is remodeled into mature bone $(6,7)$.

Blood vessels have been shown to serve an important role in bone development and fracture repair. Genetically modified mice that lack specific splice variants of vascular endothelial growth factor (VEGF)-A have blood vessel and skeletal defects (8-10). Additionally, pharmacological inhibition of angiogenesis suppresses bone formation and fracture healing $(11,12)$. Conversely, overexpression of VEGF-A in bone stimulates angiogenesis and the formation of trabecular bone (13). Although much is known about the relationship between blood vessels and bone, very little is known about the relationship between lymphatics and bone. Lymphatics are not typically present in bone (14-17). However, lymphatics form in bone and promote bone destruction in patients with Gorham-Stout disease, generalized lymphatic anomaly, or kaposiform lymphangiomatosis $(18,19)$. Additionally, work with rats has shown that bone injury induces the enlargement of draining lymph nodes (20) and studies with humans have shown that limbs with healed fractures have dilated lymphatics and enlarged lymph nodes $(21,22)$. Despite this work, it is unclear if lymphatics serve an essential role in normal bone development or repair. Therefore, we set out to characterize bone development and fracture healing in mice that have a defect in the development of the lymphatic system.

\section{MATERIALS AND METHODS}

\section{Mice and Genotyping}

The animal experiments described in this manuscript were carried out in accordance with an animal protocol approved by the Institutional Animal Care and Use Committee of UTSW. Mice were maintained in ventilated microisolator cages and were fed a standard diet ad libitum. Mice were provided igloos and nestlets as enrichment items. Prox1-tdTomato mice (23) were on a $\mathrm{C} 57 \mathrm{Bl} / 6$ genetic background and were genotyped with the following primers: 5'-GATGTGCCATAAATCCCAGA GCCTAT-3'; and 5'-CCTTGGAGCCGTACA TGAACTGG-3'. The transgenic allele was $\mathbf{4 2 0}$ bp. Vegfr3Chy mice (24) were on a $\mathrm{C} 3 \mathrm{H}+$ C57Bl/6 mixed genetic background and were genotyped with the following primers: 5'-TCC CATTTTGGAGGAAACTCAGC-3' and 5'CAAAGGTTCCTGTCACCTTGAGC-3'. The PCR product was digested overnight with BgIII. The wild-type band was approximately $250 \mathrm{bp}$ and the mutant band approximately $300 \mathrm{bp}$.

Polyethylene Glycol-Associated Solvent System (PEGASOS)

When Prox1-tdTomato mice were between 3 and 4-weeks-old, they were euthanized, then perfused with PBS + heparin and 
then with 4\% paraformaldehyde (PFA). This was done with male and female mice. Ribs were collected and fixed overnight in 4\% PFA. Samples were washed with PBS and then decalcified for 2 days with 0.5 M EDTA ( $\mathrm{pH}$, 8.0). Samples were then washed with distilled water for 30 minutes and then decolorized with $25 \%$ quardol (Sigma-Aldrich, 122262) for 1 day. Samples were delipidated with the following solutions: $30 \%$ tert-butanol $+3 \%$ quardol ( 2 hours), $50 \%$ tert-butanol $+3 \%$ quardol ( 2 hours), $70 \%$ tert-butanol $+3 \%$ quardol ( 2 hours), and $70 \%$ tert-butanol $+27 \%$ PEG-MMA-500 + 3\% quardol (overnight). Samples were then immersed in clearing medium (75\% benzyl benzoate $+23 \%$ PEGMMA-500 + 3\% quardol) for several days. Samples were stored in clearing medium until they were imaged. Samples were placed in a depression slide containing clearing medium and analyzed with a Zeiss LSM780 upright confocal/multiphoton microscope that was configured for second harmonic generation and fluorescence imaging. Imaris was used to outline Prox1-tdTomato-positive lymphatics. Imaris and FIJI (Fiji Is Just ImageJ) were also used to generate $3 \mathrm{D}$ reconstructions of bone and lymphatics.

\section{Toluidine Blue Staining}

Slides were deparaffinized, rehydrated and then stained with $0.04 \%$ Toluidine blue for 10 minutes. Stained slides were then rinsed with water, counterstained with $0.02 \%$ Fast Green for 3 minutes, rinsed with water again and then dehydrated. Coverslips were mounted with CytoSeal (Thermoscientific, 8312-4). FIJI was used to measure hypertrophic cartilage length at 6 different areas in each image.

\section{Von Kossa Staining}

Slides were deparaffinized, rehydrated and then stained with $0.1 \%$ Silver Nitrate for 30 minutes. Slides were then rinsed with water, covered with Sodium Thiosulphate for 5 minutes, rinsed with water again and then counterstained with Nuclear Fast Red for 5 minutes. Slides were then rinsed, dehydrated, and coverslipped with CytoSeal. FIJI was used to measure the Von Kossa-positive area in images.

\section{Decalcification of Bone}

Mice were euthanized and then perfused with PBS + heparin and then with $4 \%$ PFA. Tibias and surrounding muscle were fixed overnight in 4\% PFA and then decalcified for 2 weeks in $10 \%$ EDTA (pH, 7.4). Bones were then processed and embedded by the histology core at UT Southwestern Medical Center. Tissues were cut $(5 \mu \mathrm{m}$-thick) and placed on glass slides for immunohistochemistry.

\section{Immunohistochemistry}

Slides were heated at $60^{\circ} \mathrm{C}$ for 30 minutes, deparaffinized with xylene, and then rehydrated through a descending ethanol series $(100 \%-0 \%)$. A hydrogen peroxide/ methanol solution was used to block endogenous peroxidase activity and TBS $+0.2 \%$ Tween 20 (TBST) $+20 \%$ Aquablock was used to block nonspecific binding of antibodies. Slides were incubated overnight with a goat anti-Lyve1 primary antibody (R\&D Systems, \#AF2125) diluted in TBST + 5\% BSA (1:500). Slides were washed with TBST then incubated for 1 hour with an HRP-conjugated donkey anti-goat secondary antibody diluted in TBST $+5 \%$ BSA. Antibody binding was detected with an ImmPACT DAB Peroxidase Substrate Kit (Vector, \#SK-4105). Slides were then dipped in hematoxylin, dehydrated, and coverslips were mounted with CytoSeal.

\section{Evans Blue Dye Lymphangiography}

A $1 \%$ working solution of Evans blue dye was created by dissolving Evans blue dye (Sigma, \#E2129) in sterile PBS. Mice were anesthetized with an intraperitoneal injection of avertin and kept warm with a heating pad. Hind paws were then injected with Evans blue dye and approximately 3 minutes later the iliac lymph nodes were imaged.

Whole-Mount Immunofluorescence Staining 
Ears were collected, fixed overnight in 1\% PFA, washed with PBS (6 x 15 minutes), and then permeabilized with PBS $+0.3 \% \mathrm{TX}$ 100 (PBST). Nonspecific binding of antibodies was blocked with PBST $+20 \%$ Aquablock (East Coast Bio, \#PP82-W0332). Ears were incubated overnight with a goat anti-Lyve1 antibody (R\&D Systems, \#AF2125) diluted in PBST (1:1000). Tissues were washed $(3 \times 40$ minutes) with PBST and then incubated overnight with the appropriate secondary antibody diluted in PBST. Tissues were washed again ( $3 \times 40$ minutes) with PBST and then mounted on slides with ProLong Gold plus DAPI (Invitrogen, \#P36931).

\section{$\mu C T$ Analysis of Femurs}

The distal femur from each animal were imaged using a Skyscan 1072 X-ray Microtomograph (Bruker, Aartselaar, Belgium) set at $50 \mathrm{kV} / 200 \mu \mathrm{A}$ and using a $0.5 \mathrm{~mm} \mathrm{Al}$ filter. Images were obtained at $8 \mu \mathrm{m}$ pixel size with a rotation step of $0.4^{\circ}$ between each image. The $3 \mathrm{D}$ image stack was reconstructed using NRecon version 1.7.4.6 (Bruker), and the trabecular parameters; bone volume over total volume values (BV/TV), trabecular thickness (Tb.Th.), trabecular spacing (Tb.Sp.), and trabecular number (Tb.N.) were measured using methods recommended by Skyscan. The trabecular region was then defined as $\mathbf{2 0 0}$ slices offset by $\mathbf{5 0}$ slices from that reference level as recommended by Skyscan, measured using CTan software (Bruker). The segmentation process separating mineralized and nonmineralized structure was performed by drawing a region of interest (ROI) on one slice to include trabecular bone. Around 7-8 ROI were drawn at regular intervals throughout the stack. The images were then scrolled down to ensure that ROI in the remaining slices, produced by interpolation, did not include cortical and exclude trabecular region while performing trabecular bone analysis. Images were thresholded to separate bone (pixels with higher grey level) and non-bone (pixels with a lower grey level) by adjusting the grey level until most of the image noise (speckle) was removed. The threshold levels finally used in all images were 84-255.

\section{Fracture Model}

For fracture healing studies, 2-month old mice were anesthetized, and a tibia was osteotomized at the mid-shaft. Fractures were stabilized using an intramedullary pin and the wound was closed using non-absorbable sutures. This protocol is similar to previously published methods (25). Mice were imaged the day after surgery to ensure pin placement and stability, then mice were imaged weekly thereafter. For X-ray monitoring, mice were anesthetized and imaged using a UltraFocusDXA instrument (Hologic). Fracture healing was subjectively evaluated by a blinded reviewer. The blind reviewer analyzed $X$-ray images and noted the first timepoint at which a callus could be detected and the first timepoint at which a union was present between the two fractured ends.

\section{Statistical Analysis}

Data were analyzed using GraphPad Prism statistical analysis software (Version 7.0). All results are expressed as mean \pm SEM. The number of mice in each group was recorded as $n=$ number of mice. Unpaired Student's $t$-tests were performed to test means for significance. Data were considered significant at $P<0.05$.

\section{RESULTS AND DISCUSSION}

\section{The 3D Architecture of Lymphatics Around Bone}

Unlike conventional histology, new tissue clearing and imaging techniques render samples transparent and allow for an unprecedented three-dimensional view of structures (nerves, blood vessels, etc.). The polyethylene glycol-associated solvent system (PEGASOS) is a new tissue clearing method that preserves endogenous fluorescence while clearing hard tissues, such as bone (26). As the 3D architecture of bone-associated lymphatics had not been previously described, we applied the 

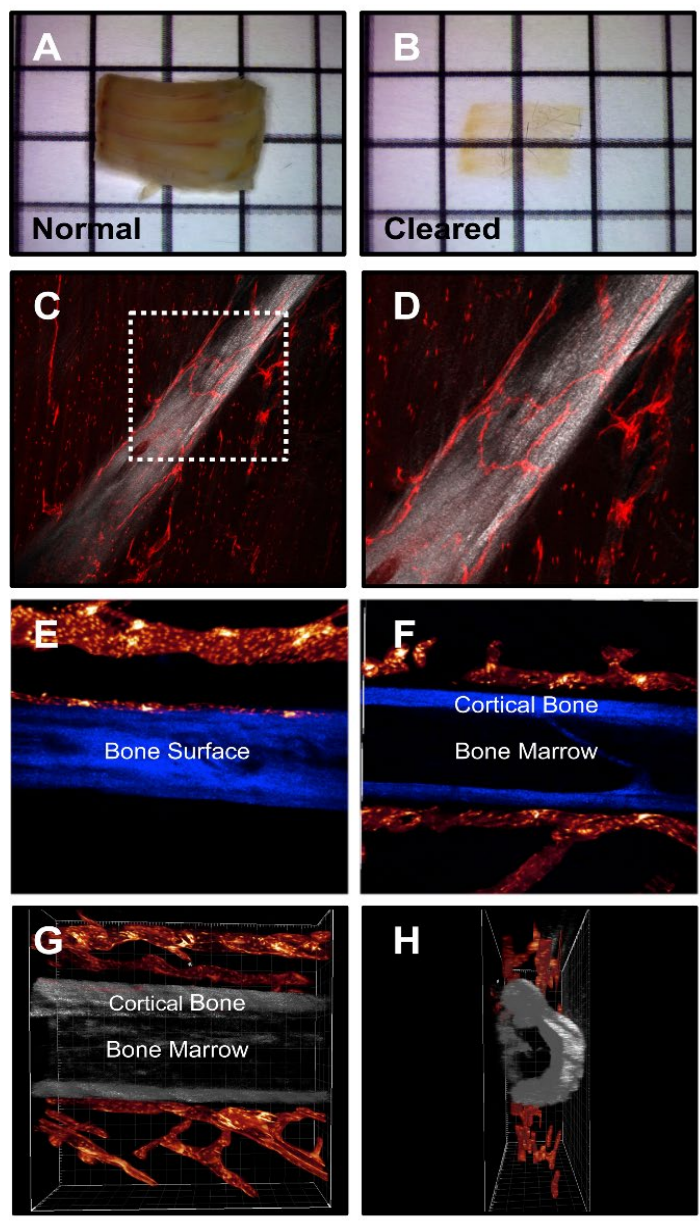

Fig. 1. Three-dimensional architecture of boneassociated lymphatics. A. Image of uncleared ribs. $B$. Image of ribs cleared by the PEGASOS method. The gridlines underneath the tissue are visible after clearing. C. Z-stack of a rib sample from a 3-weekold Prox1-tdTomato mouse. Lymphatics are colored red and bone is colored white. D. Magnified view of the boxed region in panel C. E,F. Optical sections of a rib sample from a 3-week-old Prox1-tdTomato mouse. Lymphatics are colored red and bone is colored blue. Panel E shows the bone surface and Panel F shows the marrow cavity of the same sample. $\mathrm{G}, \mathrm{H}$. Optical sections of rib sample from a 3 week-old Prox1-tdTomato mouse. Lymphatics are colored red and bone is colored white. Panel $\mathrm{H}$ is an image of a Z-stack rotated 90 degrees.

PEGASOS method to characterize the 3D architecture of bone-associated lymphatics using ribs from Prox1-tdTomato transgenic mice. These mice have fluorescent lymphatics because they express the fluorescent molecule, tdTomato, under the direction of the Prox1 promoter $(23,27)$. We chose to analyze ribs because they are thinner and flatter than long bones, making them more amendable to clearing and imaging. The PEGASOS tissue clearing method rendered ribs from Prox1tdTomato mice transparent (Fig. 1). We then imaged bone-associated lymphatics by confocal microscopy and bone by second harmonic generation. Optical sections obtained at various levels showed that lymphatics ran parallel to ribs and occasionally crisscrossed over the bones (Fig. 1). These lymphatics appeared to be initial lymphatics because they did not contain valves (Fig. 1). Importantly, bones from all Prox1-tdTomato mice $(\mathrm{n}=4 \mathrm{mice}) \mathrm{did}$ not contain lymphatics (Fig. 1). These results show that adult bones do not contain, but are surrounded by, a rich network of lymphatics.

\section{Lymphatics Are Not Present in Bone During Embryonic Development}

Although bones from adult mice do not contain lymphatics, it remains unclear whether lymphatics are present in bones during embryogenesis. To determine whether bones in embryos have lymphatics, we stained sections of humeri from E14.5, E16.5, and E18.5 embryos with an anti-Lyve1 antibody. We stained for Lyve1 because other lymphatic markers such as neuropilin-2 and podoplanin are expressed by osteoblasts and osteocytes, respectively $(15,28)$. Lyve1 was not detected in the bones at any of the embryonic timepoints studied here, suggesting that lymphatics are not present in bone during embryonic development (Fig. 2).

\section{Bone Development Occurs Normally in Vegfr $3^{\text {Chy/Chy }}$ and Vegfr $3^{\text {wt/Chy }}$ Mice}

The Chy mouse line harbors a point mutation in the kinase domain of VEGFR3. This mutation impairs kinase activity and exerts a dominant-negative effect on VEGFR3 signaling (24). Vegfr $3^{\text {Chy/Chy }}$ mice do not develop lymphatics and die shortly after birth 

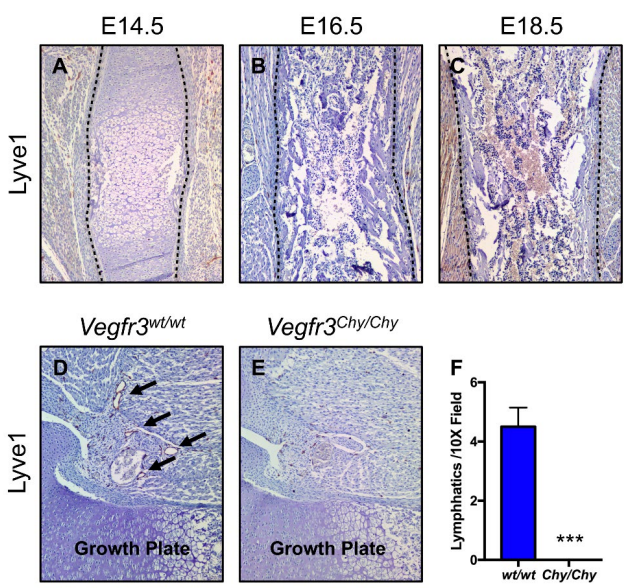

Fig. 2. Lymphatics are not in bone during embryonic development. Images of humeri from $E 14.5(A ; n=3), E 16.5(B ; n=3)$ and $E 18.5(C ; n=3)$ embryos stained with an anti-Lyve1 antibody. Lymphatics were in periosseous muscle tissue but not in cortical bone or the marrow cavity. D,E. Lyve1 immunostaining results for humeri from E16.5 Vegfr $3^{\mathrm{wt} / \mathrm{wt}}$ and Vegfr3 $3^{\mathrm{Chy} / \mathrm{Chy}}$ embryos. F. Vegfr3 $3^{\mathrm{wt} / \mathrm{wt}}$ embryos $(4.5 \pm 0.6455, n=4)$ had significantly more lymphatics than Vegfr $3^{\text {Chy/Chy }}$ embryos $(0 \pm 0, n=4)$. *** $P<0.001$; unpaired Student's t-test.

$(29,30)$. In contrast, Vegfr $3^{\text {wt/Chy }}$ mice are viable and have significantly fewer lymphatics than wild-type mice (24). To verify that Vegfr $3^{\text {Chy/Chy }}$ mice do not develop lymphatics, we stained humeri from E16.5 embryos for Lyve1. We found that Vegfr $3^{w t / w t}$ embryos had lymphatics in periosseous muscle, while lymphatics were not detected in Vegfr3 $3^{\text {Chy/Chy }}$ embryos (Fig. 2).

To determine whether lymphatics are required for proper bone development, we analyzed humeri from E16.5 and E18.5 Vegfr $3^{\text {Chy/Chy }}$ embryos. The overall architecture of bones from Vegfr $3^{C h y / C h y}$ embryos appeared normal by H\&E staining (Fig. 3). To characterize the morphology of the growth plate, we stained sections with toluidine blue and found that the growth plate, including the zone of hypertrophic cartilage, appeared normal in Vegfr $3^{\text {Chy/Chy }}$ embryos (Fig. 3). Next, we assessed mineralized bone by Von Kossa staining. The bone collar was present and appeared normal in Vegfr $3^{\text {Chy/Chy }}$ embryos, and the Von Kossa-positive area was not significantly different between Vegfr $3^{w / w t}$ and

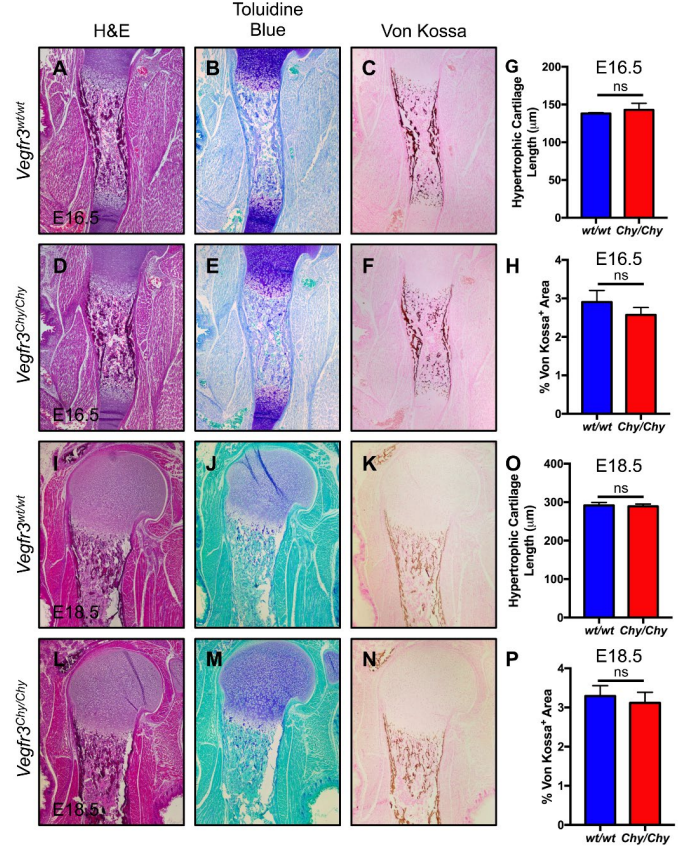

Fig. 3. Bone development is normal in Vegfr $3^{\text {Chy/Chy }}$ embryos. A-F. Hematoxylin and eosin $(A, D)$, toluidine blue $(B, E)$, and Von Kossa $(C, F)$ staining of humeri from E16.5 Vegfr $3^{\mathrm{wt} / \mathrm{wt}}$ and Vegfr $3^{\text {Chy/Chy }}$ embryos. G. The length of hypertrophic cartilage was not significantly different between $E 16.5 \mathrm{Vegfr}^{\mathrm{wt} / \mathrm{wt}}(138.2 \pm 1.087, \mathrm{n}=4)$ and Vegfr3 $3^{\text {Chy/Chy }}(143 \pm 8.651, n=4)$ embryos. $H$. The percent of Von Kossa-positive area was not significantly different between E16.5 Vegfr $3^{\text {wt/wt }}(2.905 \pm$ $0.2992, n=4)$ and Vegfr3 $3^{\text {Chy/Chy }}(2.57 \pm 0.1947, n=4)$ embryos. I-N. Hematoxylin and eosin $(I, L)$, toluidine blue $(J, M)$, and Von Kossa $(K, N)$ staining of humeri from E18.5 Vegfr $3^{\text {wt/wt }}$ and Vegfr $3^{\text {Chy/Chy }}$ embryos. $O$. The length of hypertrophic cartilage was not significantly different between E18.5 Vegfr ${ }^{\mathrm{wt} / \mathrm{wt}}(291.5 \pm$ $7.73, n=4)$ and Vegfr $3^{\text {Chy/Chy }}(289 \pm 6.028, n=3)$ embryos. $P$. The percent of Von Kossa-positive area was not significantly different between E18.5 Vegfr3 $^{\mathrm{wt} / \mathrm{wt}}(3.293 \pm 0.2662, n=4)$ and Vegfr3 $3^{\text {Chy/Chy }}$ $(3.117 \pm 0.2703, n=3)$ embryos. $n s=$ not significant; unpaired Student's t-test.

Vegfr3 $3^{\text {Chy/Chy }}$ embryos (Fig. 3).

We then sought to determine whether a reduction in lymphatics affected postnatal bone development. We first confirmed that the Von Kossa-positive area was not significantly different between Vegfr $3^{\text {wt/wt }}$ and Vegfr $3^{\text {Chy/Chy }}$ embryos (Fig. 3). 

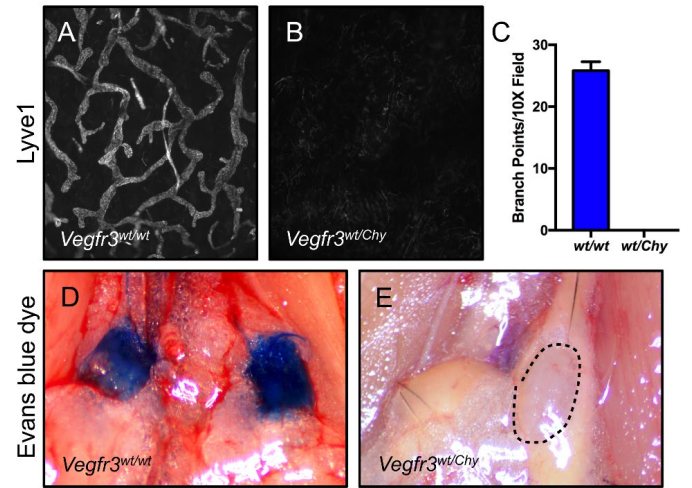

Fig. 4. Lymphatic development and function are impaired in Vegfr $3^{\text {wt/Chy }}$ mice. A,B. Representative images of ear skin whole-mounts immunostained for Lyve1. C. Vegfr $3^{\mathrm{wt} / \mathrm{wt}}$ mice $(25.8 \pm 1.463, \mathrm{n}=5)$ had significantly more lymphatic branch points than Vegfr3wt/Chy mice $(0 \pm 0, n=3) . P<0.0001$; unpaired Student's t-test. D,E. Evans blue dye was transported from the hind paws to the iliac lymph nodes in Vegfr $3^{\mathrm{wt} / \mathrm{wt}}$ mice ( $\mathrm{n}=4$ mice), but not in Vegfr $3^{\mathrm{wt} / \mathrm{Chy}}$ mice ( $\mathrm{n}=6$ mice). An iliac lymph node in a Vegfr $3^{\mathrm{wt} / \mathrm{Chy}}$ mouse that is not filled with Evans blue dye is outlined in panel $E$.

We then sought to determine whether a reduction in lymphatics affected postnatal bone development. We first confirmed that Vegfr $3^{w t / C h y}$ mice have fewer lymphatics than Vegfr $3^{\text {wt/wt }}$ mice by whole-mount immunofluorescence staining of ear skin (Fig. 4). We also confirmed that lymph transport is altered in Vegfr $3^{\text {wt/Chy }}$ mice by Evans blue dye lymphangiography (Fig. 4). After verifying that the lymphatic network was altered in Vegfr $3^{\text {wt } / \text { Chy }}$ mice, we analyzed femurs from 8-week-old Vegfr $3^{\text {wt/Chy }}$ mice by $\mu \mathrm{CT}$. We found that the bone volume over total volume (BV/TV), trabecular thickness (Tb.Th.), trabecular spacing (Tb.Sp.), and trabecular number (Tb.N.) were not significantly different between Vegfr $3^{w / w t}$ and Vegfr $3^{w / / C h y}$ mice (Fig. 5). Together, these results suggest that lymphatics do not serve an essential function in embryonic or postnatal bone development.

\section{Fracture Healing Is Normal in Vegfr $3^{\text {wt/Chy }}$ Mice}

Anti-lymphangiogenic drugs or hypo-
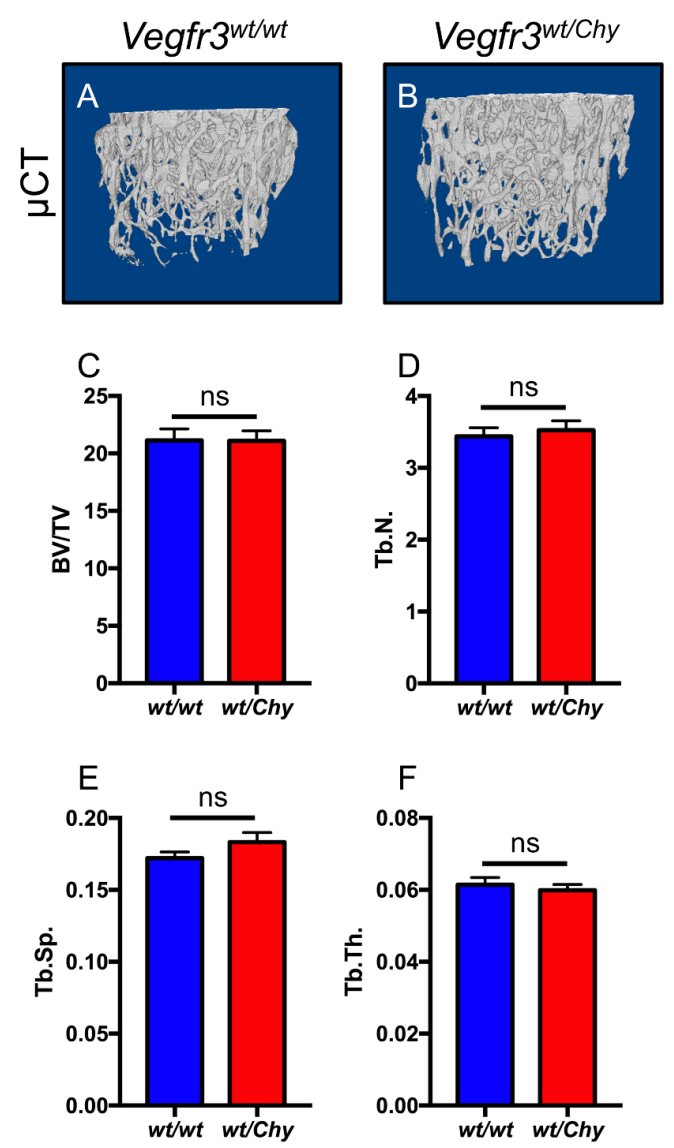

Fig. 5. Trabecular bone is normal in adult Vegfr $3^{\text {wt/Chy }}$ mice. $A, B$. $\mu C$ T images of trabecular bone in 8 -week-old Vegfr $3^{\text {w//wt }}$ and Vegfr $3^{\text {wt/Chy }}$ male mice. $C . B V / T V$ values were not significantly different between Vegfr $3^{\mathrm{w} / \mathrm{wt}}(21.14 \pm 0.9831, n=10)$ and Vegfr $3^{\text {wt/Chy }}(21.09 \pm 0.873, n=9)$ mice. $D$. Trabecular number values were not significantly different between Vegfr $3^{\mathrm{wt} / \mathrm{wt}}(3.439 \pm 0.1192, n=10)$ and Vegfr $3^{\text {w/Chy }}(3.524 \pm 0.1302, n=9)$ mice. $E$. Trabecular spacing values were not significantly different between $\operatorname{Vegfr}{ }^{\mathrm{wt} / \mathrm{wt}}(0.1721 \pm 0.004336$, $n=10)$ and $\mathrm{Vegfr} 3^{\mathrm{w} / \mathrm{Chy}}(0.1833 \pm 0.006563, n=9)$ mice. F. Trabecular thickness values were not significantly different between Vegfr $3^{\mathrm{w} / \mathrm{wt}}(0.06146 \pm$ $0.001954, n=10)$ and Vegfr $3^{\text {wt/Chy }}(0.05991 \pm$ $0.001584, n=9)$ mice. $n s=$ not significant; unpaired Student's t-test.

plasia of the lymphatic system can impair the resolution of inflammation (31). Importantly, chronic inflammation impairs fracture healing (32). To determine whether lymphatic hypo- 


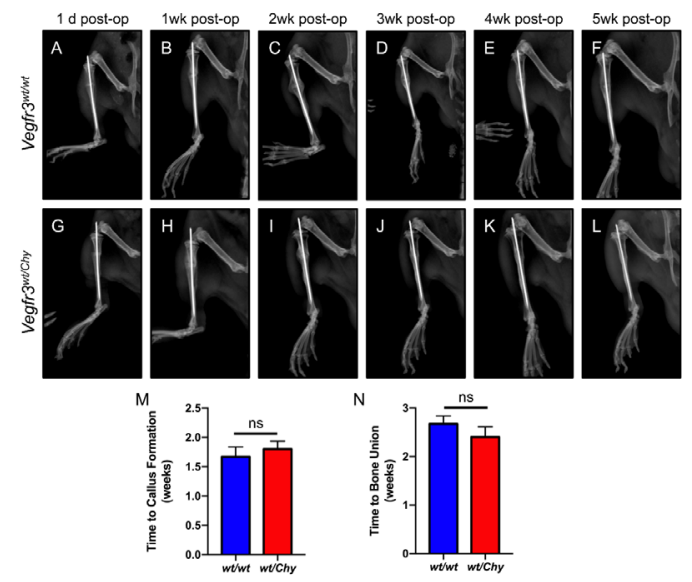

Fig. 6. Time to callus formation is normal in male Vegfr $3^{\mathrm{wt} / \mathrm{Chy}}$ mice. A-L. X-rays of fractures in male

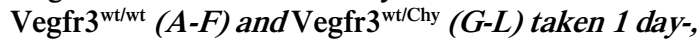
1 week-, 2 weeks-, 3 weeks-, 4 weeks-, and 5 weekspost injury. Fractures were induced when mice were 8 -weeks-old. M. The time to callus formation was similar between Vegfr3 ${ }^{\mathrm{wt} / \mathrm{wt}}(1.67$ weeks \pm 0.1667 , $n=9)$ and Vegfr $3^{\text {wt/Chy }}(1.8$ weeks $\pm 0.1328, n=10)$ mice. $N$. The time to bone union was similar between Vegfr $3^{\mathrm{wt} / \mathrm{wt}}(2.67 \pm 0.1667, n=9)$ and Vegfr $3^{\text {wt/Chy }}(2.4 \pm 0.2119, n=10)$ mice. $n s=n o t$ significant; unpaired Student's t-test.

plasia impairs fracture healing, we induced tibia fractures in 8-week old Vegfr $3^{w t / w t}$ and Vegfr $3^{\text {wt/Chy }}$ mice. Radiographs were evaluated weekly to monitor the healing process in Vegfr $3^{w t / w t}$ and Vegfr $3^{w t / C h y}$ mice (Figs. 6 and 7 ). This revealed that the time to callus formation and bone union was similar between Vegfr $3^{\text {wt/wt }}$ and Vegfr $3^{w t / C h y}$ mice (Figs. 6 and 7). This was true for female and male mice, suggesting that lymphatic hypoplasia does not affect fracture repair.

\section{CONCLUSIONS}

The relationship between blood vessels and bone has been extensively studied. In contrast, the relationship between lymphatics and bone is poorly understood. In the present study, we show that bone development is normal in Vegfr $3^{\text {Chy/Chy }}$ embryos and Vegfr $3^{\text {wt/Chy }}$ mice. We also show that fracture healing is normal in Vegfr $3^{\text {wt/Chy }}$ mice. It is possible that the remaining lymphatics in Vegfr $3^{\text {w/Chy }}$ mice

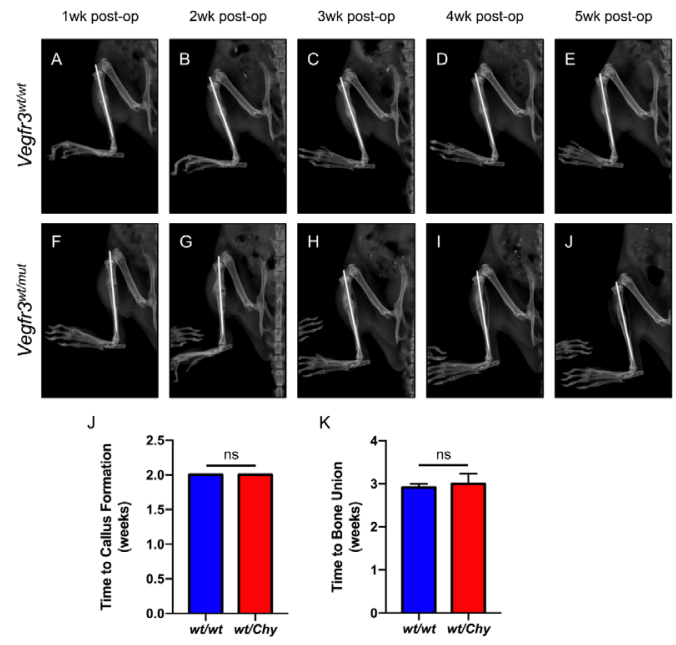

Fig. 7. Time to callus formation is normal in female Vegfr $3^{\mathrm{wt} / \mathrm{Chy}}$ mice. $A-J$. X-rays of fractures in female Vegfr3wt/wt (A-E) and Vegfr3wt/Chy (F-J) taken 1 week-, 2 weeks-, 3 weeks-, 4 weeks-, and 5 weeks-post injury. Fractures were induced when mice were 8 -weeks-old. $K$. The time to callus formation was similar between Vegfr $3^{w t / w t}(2 \pm 0.0, n=12)$ and Vegfr $3^{\mathrm{wt} / \mathrm{Chy}}(2 \pm 0.0, n=12)$ mice. L. The time to bone union was similar between Vegfr $3^{\mathrm{wt} / \mathrm{wt}}(2.916 \pm$ $0.08314, n=12)$ and Vegfr $3^{\text {wt/Chy }}(3 \pm 0.2356, n=12)$ mice. $n s=$ not significant; unpaired Student's t-test.

are sufficient to promote normal fracture healing. In the future, different animal models could be used to determine whether a complete absence of lymphatics affects fracture healing. In conclusion, although lymphatics promote bone loss in diseases such as Gorham-Stout disease, generalized lymphatic anomaly, and kaposiform lymphangiomatosis, our results suggest that they do not play a major role in normal bone development or repair.

\section{ACKNOWLEDGMENTS}

The authors would like to thank Rolf Brekken and David Primm for help in editing the manuscript. This work was supported by a grant from the NIH 1R01HL144793-01 (MTD) and by Scottish Rite for Children (JJR). The C3H101H-Flt4Chy/H (VEGFR3) mice were obtained from the MRC Harwell which distributes this strain on behalf of the European Mouse Mutant Archive (EMMA: 
www.infrafrontier.eu). The repository number is EM:00068. The $\mathrm{C} 3 \mathrm{H} 101 \mathrm{H}-\mathrm{Flt} 4 \mathrm{Chy} / \mathrm{H}$ (VEGFR3) mice were originally generated at the MRC-Harwell.

Authors AK McCarter and A Khalid as well as authors JJ Rios and MT Dellinger contributed equally to this work.

\section{CONFLICT OF INTEREST AND DISCLOSURE}

The authors declare no competing financial interests exist.

\section{REFERENCES}

1. Burge, R, B Dawson-Hughes, DH Solomon, et al: Incidence and economic burden of osteoporosisrelated fractures in the United States, 2005-2025. J. Bone Miner Res. 22 (2007), 465-475.

2. Calori, GM, EL Mazza, S Mazzola, et al: Nonunions. Clin. Cases Miner Bone Metab. 14 (2017), 186-188.

3. Watson, EC, RH Adams: Biology of bone: The vasculature of the skeletal system. Cold Spring Harb. Perspect Med. 8 (2018), a031559.

4. $\mathrm{Hu}, \mathrm{K}, \mathrm{BR}$ Olsen: Vascular endothelial growth factor control mechanisms in skeletal growth and repair. Dev. Dyn. 246 (2017), 227-234.

5. Kronenberg, HM: Developmental regulation of the growth plate. Nature 423 (2003), 332-336.

6. Marsell, R, TA Einhorn: The biology of fracture healing Injury. 42 (2011), 551-555.

7. Loi, F, LA Cordova, J Pajarinen, et al: Inflammation, fracture and bone repair. Bone 86 (2016), 119-130.

8. Maes, C, P Carmeliet, K Moermans, et al: Impaired angiogenesis and endochondral bone formation in mice lacking the vascular endothelial growth factor isoforms VEGF164 and VEGF188. Mech. Dev. 111 (2002), 61-73.

9. Zelzer, E, W McLean, YS Ng, et al: Skeletal defects in VEGF(120/120) mice reveal multiple roles for VEGF in skeletogenesis. Development 129 (2002), 1893-1904.

10. Maes, C, I Stockmans, K Moermans, et al: Soluble VEGF isoforms are essential for establishing epiphyseal vascularization and regulating chondrocyte development and survival. J. Clin. Invest. 113 (2004), 188-199.

11. Gerber, HP, TH Vu, AM Ryan, et al: VEGF couples hypertrophic cartilage remodeling, ossification and angiogenesis during endochondral bone formation. Nat. Med. 5 (1999), 623-628.

12. Hausman, MR, MB Schaffler, RJ Majeska: Prevention of fracture healing in rats by an inhibitor of angiogenesis. Bone 29 (2001), 560-564.

13. Maes, C, S Goossens, S Bartunkova, et al:
Increased skeletal VEGF enhances beta-catenin activity and results in excessively ossified bones. EMBO J. 29 (2010), 424-441.

14. Edwards, JR, K Williams, LG Kindblom, et al: Lymphatics and bone. Hum. Pathol. 39 (2008), 4955.

15. Hominick, D, A Silva, N Khurana, et al: VEGF-C promotes the development of lymphatics in bone and bone loss. Elife 7 (2018), e34323.

16. Monroy, M, AL McCarter, D Hominick, et al: Lymphatics in bone arise from preexisting lymphatics. Development 147 (2020), dev184291.

17. Rodriguez-Laguna, L, N Agra, K Ibanez, et al: Somatic activating mutations in PIK3CA cause generalized lymphatic anomaly. J. Exp. Med. 216 (2019), 407-418.

18. Dellinger, MT, N Garg, BR Olsen: Viewpoints on vessels and vanishing bones in Gorham-Stout disease. Bone 63 (2014), 47-52.

19. Croteau, SE, HP Kozakewich, AR Perez-Atayde, et al: Kaposiform lymphangio-matosis: A distinct aggressive lymphatic anomaly. J. Pediatr. 164 (2014), 383-388.

20. Szczesny, G, WL Olszewski, M Zaleska: Limb lymph node response to bone fracture. Lymphat. Res. Biol. 2 (2004), 155-164.

21. Szczesny, G, WL Olszewski, A Gorecki: Lymphoscintigraphic monitoring of the lower limb lymphatic system response to bone fracture and healing. Lymphat. Res. Biol. 3 (2005), 137-145.

22. Szczesny, G, WL Olszewski, M Gewartowska, et al: The healing of tibial fracture and response of the local lymphatic system. J. Trauma 63 (2007), 849854.

23. Yanev, P, K Poinsatte, D Hominick, et al: Impaired meningeal lymphatic vessel development worsens stroke outcome. J. Cereb. Blood Flow Metab. 40 (2020), 263-275.

24. Karkkainen, MJ, A Saaristo, L Jussila, et al: A model for gene therapy of human hereditary lymphedema. Proc. Natl. Acad. Sci USA 98 (2001), 12677-12682.

25. Schindeler, A, A Morse, L Harry, et al: Models of tibial fracture healing in normal and Nf1-deficient mice. J. Orthop. Res. 26 (2008), 1053-1060.

26. Jing, D, S Zhang, W Luo, et al: Tissue clearing of both hard and soft tissue organs with the PEGASOS method. Cell Res. 28 (2018), 803-818.

27. Kontarakis, Z, A Rossi, S Ramas, et al: Mir-126 is a conserved modulator of lymphatic development. Dev. Biol. 437 (2018), 120-130.

28. Verlinden, L, C Kriebitzsch, I Beullens, et al: Nrp2 deficiency leads to trabecular bone loss and is accompanied by enhanced osteoclast and reduced osteoblast numbers. Bone 55 (2013), 465-475.

29. Jakus, Z, JP Gleghorn, DR Enis, et al: Lymphatic function is required prenatally for lung inflation at birth. J. Exp. Med. 211 (2014), 815-826.

30. Zhang, L, F Zhou, W Han, et al: VEGFR-3 ligandbinding and kinase activity are required for lymphangiogenesis but not for angiogenesis. Cell Res. 20 (2010), 1319-1331. 
31. Abouelkheir, GR, BD Upchurch, JM Rutkowski: Lymphangiogenesis: Fuel, smoke, or extinguisher of inflammation's fire? Exp. Biol. Med. (Maywood). 242 (2017), 884-895.

32. Claes, L, S Recknagel, A Ignatius: Fracture healing under healthy and inflammatory conditions. Nat. Rev. Rheumatol. 8 (2012), 133-143.

Michael T. Dellinger, PhD

Department of Molecular Biology and the Hamon Center for Regenerative Science and Medicine

UT Southwestern Medical Center

Dallas, TX, USA

Email: michael.dellinger@utsouthwestern.edu

Jonathan J. Rios, PhD

Scottish Rite for Children

UT Southwestern Medical Center

Dallas, TX USA

Email: jonathan.rios@tsrh.org 